\title{
Visualizing stages of cortical atrophy in progressive MCI from the ADNI cohort
}

\author{
S.F. Eskildsen ${ }^{1,2}$, V. Fonov ${ }^{2}$, P. Coupé ${ }^{2,3}$, D. L. Collins ${ }^{2}$ and the Alzheimer's Disease Neuroimaging Initiative * \\ ${ }^{1}$ Center of Functionally Integrative Neuroscience, Aarhus University, Aarhus, Denmark \\ ${ }^{2}$ McConnell Brain Imaging Centre, Montréal Neurological Institute, McGill University, Montréal, Canada \\ ${ }^{3}$ Laboratoire Bordelais de Recherche en Informatique, Unité Mixte de Recherche CNRS (UMR 5800), 351 \\ cours de la Libération F-33405 Talence cedex
}

* Data used in the preparation of this article were obtained from the Alzheimer's Disease Neuroimaging Initiative (ADNI)
database (www.loni.ucla.edu/ADNI). As such, the investigators within the ADNI contributed to the design and
implementation of ADNI and/or provided data but did not participate in analysis or writing of this report. A complete listing
of ADNI investigators can be found at: http://loni.ucla.edu/ADNI/Collaboration/ADNI_Authorship_list.pdf

Learning objectives: To investigate the cortical pattern of atrophy at different stages in patients with mild cognitive impairment $(\mathrm{MCl})$, which progress to clinically definite Alzheimer's disease.

Topic area: Neuroimaging - Early detection and tracking

Keywords: ADNI, Alzheimer's disease, MCI, MRI, cortical thickness, prediction

Background: Amnestic mild cognitive impairment $(\mathrm{MCl})$ is considered a condition where patients are at risk of developing clinically definite Alzheimer's disease (AD) with an annual conversion rate of approximately $15 \%[1]$. AD is characterized by progressive brain atrophy with major impact on the cerebral cortex and medial temporal lobe structures such as hippocampus. Understanding the structural pattern of cortical atrophy at different stages of $\mathrm{MCl}$, before $A D$ can be diagnosed, may help in patient monitoring and prognosis. We used data from the Alzheimer's Disease Neuroimaging Initiative (ADNI) to calculate and visualize the cortical atrophy at different stages in patients who eventually converted to clinically definite AD.

Methods: We selected patients with a diagnosis of $\mathrm{MCI}$ from the ADNI database who converted to $A D$ during the follow-up period. T1-weighted MRI scans were collected at time of conversion( $n=140)$ and at intervals prior to the date of conversion: 6 months( $n=121), 12$ months( $n=128), 24$ months( $n=62)$ and 36 months $(n=29)$. As control group we collected all baseline scans of healthy controls $(H C)(n=226)$. Images were denoised[2], bias field corrected[3], linearly co-registered[4], skull-stripped[5], and cortical thickness were measured using FACE[6] and mapped to an average cortical surface. Statistical maps of differences in cortical thickness between groups of $\mathrm{MCl}$ patients and $\mathrm{HC}$ were constructed and corrected for multiple comparisons.

Results: Three years prior to clinically definite $A D$, the $M C l$ patients show signs of atrophy in the parahippocampal gyrus, the cingulate gyrus and temporal lobes (figure). At two years before conversion, atrophy has spread to large parts of the temporal and frontal lobes, much of the cingulate gyrus and parts of the orbitofrontal region. Twelve months before conversion, atrophy is present over all the cortex except 
the occipital lobe and the sensory-motor areas. Six months later and at conversion this pattern persists, but with more severe atrophy.

Conclusions: Focal atrophy can be observed in patients with $\mathrm{MCl}$ three years before they are diagnosed with clinically definite $\mathrm{AD}$. The atrophy accelerates during the $\mathrm{MCl}$ stage and affects the entire cortex except the occipital and sensory-motor cortex at the time of diagnosis - not unlike the progressive patterns of amyloid deposition described by Braak[7]. These patterns may be used to assess the disease progression in patients with $\mathrm{MCl}$.

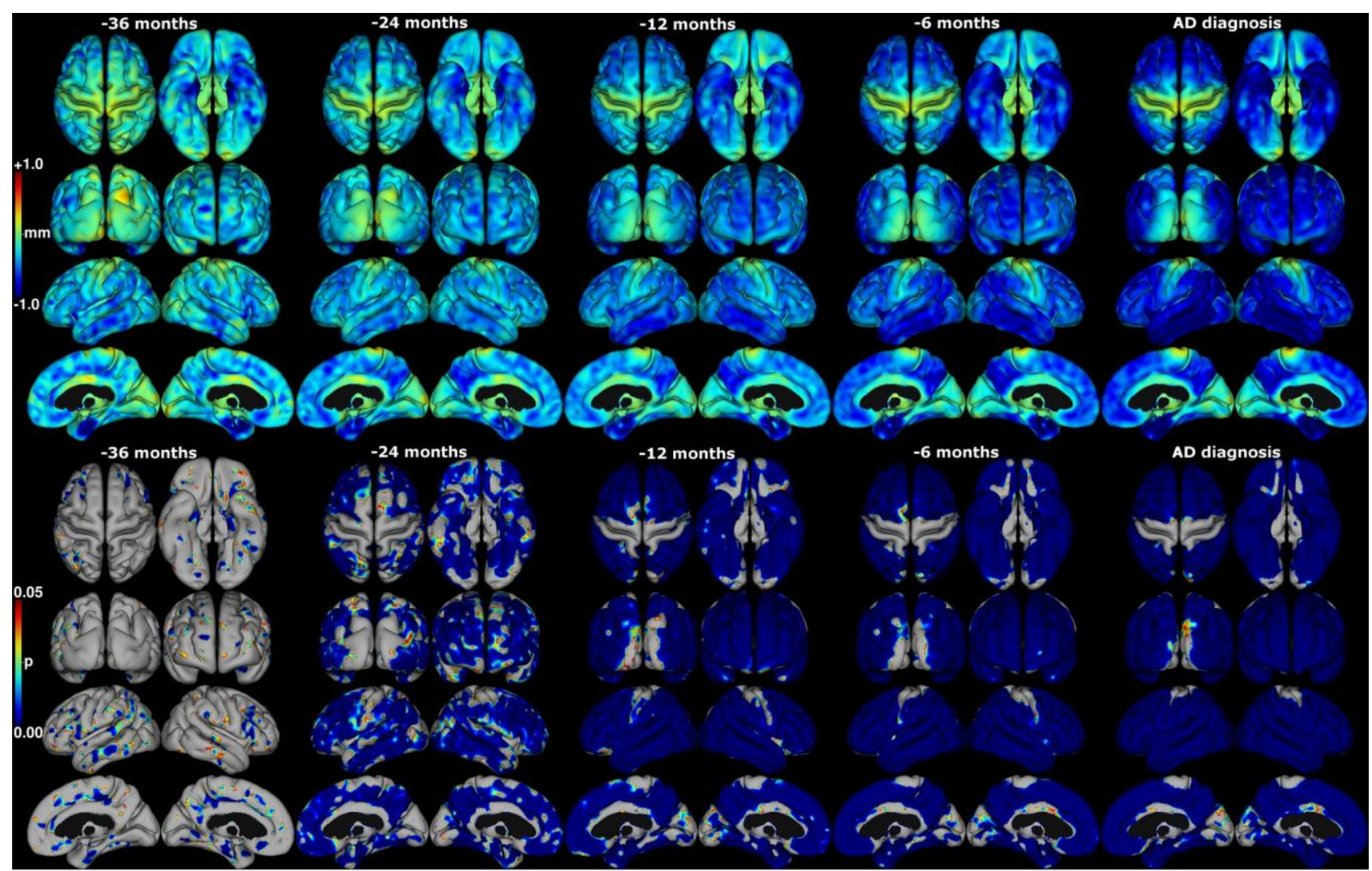

Cortical thickness statistics of MCI patients compared to healthy controls at intervals before AD diagnosis and at time of diagnosis. Top row: differences in mean cortical thickness. Bottom row: statistical maps of differences in cortical thickness. FDR corrected p-maps.

\section{References:}

[1] Petersen, R.C., et al. (2001) Current concepts in mild cognitive impairment. Arch Neurol 58, 1985-1992.

[2] Coupé, P., et al. (2008), 'An Optimized Blockwise NonLocal Means Denoising Filter for 3-D Magnetic Resonance Images'. IEEE Transactions on Medical Imaging, vol. 27, no. 4, pp. 425-441.

[3] Sled, J.G., et al. (1998), 'A nonparametric method for automatic correction of intensity nonuniformity in MRI data', IEEE Transactions on Medical Imaging, vol. 17, no. 1, pp. 87-97.

[4] Collins, D.L., et al. (1994), 'Automatic 3D intersubject registration of MR volumetric data in standardized Talairach space' Journal of Computer Assisted Tomography, vol. 18, no. 2, pp. 192-205.

[5] Eskildsen, S.F., et al. (2012), 'BEaST: Brain Extraction based on nonlocal Segmentation Technique', Neurolmage, vol. 59, no. 3, pp. 2362-2373. 
[6] Eskildsen, S.F., et al. (2006), 'Active surface approach for extraction of the human cerebral cortex from MRI', Lecture Notes in Computer Science, vol. 4191, pp. 823-830.

[7] Braak, H \& Braak E, (1991), 'Neuropathological staging of Alzheimer-related changes', Acta Neuropathologica, 82:239-259. 